\title{
BMJ Open Protocol for a systematic review of psychological treatment for methamphetamine use: an analysis of methamphetamine use and mental health symptom outcomes
}

\author{
Alexandra Stuart, ${ }^{1}$ Amanda L Baker, ${ }^{2}$ Jenny Bowman, ${ }^{1}$ Kristen McCarter, ${ }^{2}$ \\ Alexandra Mary Janice Denham, ${ }^{2}$ Nicole Lee, ${ }^{3}$ Kim Colyvas, ${ }^{4}$ Adrian Dunlop ${ }^{2}$
}

To cite: Stuart A, Baker AL, Bowman J, et al. Protocol for a systematic review of psychological treatment for methamphetamine use: an analysis of methamphetamine use and mental health symptom outcomes. BMJ Open 2017;7:e015383. doi:10.1136/ bmjopen-2016-015383

- Prepublication history and additional material are available. To view please visit the journal online (http://dx.doi.org/10. 1136/bmjopen-2016-015383).

Received 30 November 2016 Revised 2 June 2017 Accepted 30 June 2017

CrossMark

${ }^{1}$ School of Psychology, Faculty of Science, University of Newcastle, Callaghan, New South Wales, Australia

${ }^{2}$ School of Medicine and Public Health, Faculty of Health and Medicine, University of Newcastle, Newcastle, New South Wales, Australia

${ }^{3}$ Faculty of Health Sciences, Curtin University, Bentley, Australia

${ }^{4}$ School of Mathematical and Physical Sciences, Faculty of Science, University of Newcastle, Callaghan, New South Wales, Australia

Correspondence to Alexandra Stuart; alexandra.m.stuart@uon.edu.au

\section{ABSTRACT}

Introduction People who use methamphetamine (MA) regularly, often experience symptoms of mental ill health associated with the use of the drug. These include symptoms of psychosis, depression, anxiety and also cognitive deficits. Accordingly, psychological treatments aim to reduce MA use and related problems, including symptoms of mental ill health. Although there has been a substantial body of research reporting on the evidence of effectiveness of psychological treatments for MA use, there is a paucity of research addressing the effectiveness of these treatments for coexisting symptoms of mental ill health. We aim to address this gap by providing a comprehensive overview of the evidence for psychological treatments for MA use and associated symptoms of mental ill health in experimental/controlled clinical studies. In addition, a critical evaluation of study methods and the outcomes of psychological interventions on MA use and symptoms of mental ill health will be conducted.

Methods and analysis The Cochrane Handbook for Systematic Reviews of Interventions and the Preferred Reporting Items for Systematic Reviews and MetaAnalysis statement will be used to inform the methods of this review. Eight electronic peer-reviewed databases will be searched. Pilot searches have been conducted for MA literature considering controlled clinical trials only. Eligible articles will be independently assessed against inclusion criteria. Before final analyses are completed, searches will be rerun and if eligible, additional studies will be retrieved for inclusion. A quantitative synthesis of the findings will be reported where possible, and 'summary of findings' tables will be generated for each comparison. Risk ratios and $95 \% \mathrm{Cl}$ (dichotomous outcomes) will be calculated and/or effect size according to Cohen's formula (continuous outcomes) for the primary outcome of each trial.

Ethics and dissemination No ethical issues are foreseen. Findings will be disseminated widely to clinicians and researchers via journal publication and conference presentation(s).

Trial registration number CRD42016043657.

\section{Strengths and limitations of this study}

- The first systematic review of interventions for methamphetamine (MA) use and associated mental health outcomes.

- This systematic review will reduce and protect against risk of bias.

- If a meta-analysis is possible, this can detect small but clinically relevant effects of interventions to reduce MA use and improve $\mathrm{MH}$ outcomes.

- Most trials have been conducted in Western countries, so generalisability of findings to other contexts is unknown.

- If a meta-analysis is appropriate, conducting this form of analysis can mean that an assumption is made about the methodology of interventions as being consistent across studies.

\section{INTRODUCTION}

\section{Rationale}

Methamphetamine (MA) is a psychostimulant that when used regularly is associated with harms such as injecting and sexual risk-taking behaviour, symptoms of mental ill health (eg, psychosis, aggression, depression and/or anxiety), psychomotor, social and cognitive impairment, criminal activity and sometimes death caused by overdose. ${ }^{1-7}$ MA use can be considered problematic if an individual continues the use of the drug despite experiencing significant harms. ${ }^{8}$ Increased production and availability of the drug has influenced regular use, consequently increasing drug-related harms. ${ }^{7}$

Substance use accounts for an increasing proportion of the global burden of disease. ${ }^{9}$ Amphetamine-type stimulants have become the most prevalent type of psychostimulants used in the world, and it is estimated that there are 24 million users worldwide. ${ }^{10}$ In 
South Asia and the Middle East, MA is becoming increasingly popular, and use is already well established in the USA, Australia, China, Mexico and Thailand..$^{10}{ }^{11}$ Due to the drug's psychological and medical impact, the detrimental effects can be seen in entire communities, whole populations and individual users. ${ }^{10}$

The disease burden in Australia attributable to MA is of international relevance as Australia has reputable data for mortality rates, and the rates of illicit drug use are similar to those in other high-income countries. ${ }^{11}$ In recent years, a more potent form of MA (crystal) has increased in popularity across the globe and particularly in Asia and Australia. ${ }^{12}$ Smoking crystal MA is the most popular form of use in Australia, followed by injection..$^{10}$ Although MA was used by only $2 \%$ of Australian adults in the 12 months to $2013,{ }^{12}$ the use of crystal MA had doubled since 2010 from $22 \%$ to $50 \%$. In addition, the proportion of people using daily or weekly increased from $12 \%$ to $25 \%$ over the same period. ${ }^{12}$

The shift to crystal MA use in Australia has seen mental health problems associated with this form of the drug substantially worsen. From 2009 to 2014, the annual total number of mental health presentations, overdose and drug and alcohol presentations at NSW public hospital emergency departments related to MA use increased more than sevenfold, from 394 to $296 .^{13}$ This rise in mental health presentations associated with crystal MA use has led to increased interest in mental health treatment outcomes following treatment for MA use.

Psychostimulants are a unique group of substances because they are more likely to induce psychosis than other illicit drugs. ${ }^{14}$ Although vulnerability to psychotic symptoms differs among people who use MA, these symptoms are more apparent in people who use MA on a regular basis. ${ }^{14}$ In addition, depression, anxiety, suicidal ideation, dysphoria and cognitive deficits have been commonly reported to co-occur in people using MA. ${ }^{15}{ }^{16}$ McKetin et $a l^{17}$ reported that a quarter of people using MA in their cross-sectional study experienced severe disability in their psychological functioning. Glasner-Edwards $e t a l^{8}$ identified that people who experience depressive symptoms and use MA may have a poorer prognosis for both conditions and may experience worse treatment outcomes. Furthermore, Newton et $a l^{19}$ suggested that depressive symptoms may contribute to negative reinforcement and more frequent use of MA, consequently impacting on psychological treatment outcomes. In summary, it appears that MA use is associated with an array of psychological difficulties that may affect one's response to treatment. ${ }^{20}$

Psychological treatment, incorporating elements such as psychotherapy, psychoeducation and relapse prevention are major treatments for MA use that focus on abstinence and reducing symptoms of mental ill health. Treatment retention can be difficult for MA users in psychological treatment. ${ }^{21}$ Pharmacotherapies have been used in conjunction with the aim of improving treatment engagement and retention; ${ }^{21}$ however, research on the efficacy of pharmacotherapies to date has produced inconsistent results. ${ }^{22}$ There is no evidence to suggest that agonist drug treatments can reduce psychological distress associated with MA use. ${ }^{23}{ }^{24}$ In a review conducted by Elkashef $e t a l,{ }^{25}$ pharmacotherapies such as bupropion and sertraline did not increase abstinence from MA in groups who received this drug compared with controls. ${ }^{26}$ The evidence base for pharmacological approaches for MA use is limited, with conflicting evidence for medication approaches, for withdrawal, maintenance or relapse prevention treatment. ${ }^{27}$

In line with the psychological and public health implications of MA use, there has been a considerable amount of research on psychological treatments for treating MA use and withdrawal. ${ }^{28}$ Thus, psychological treatment is the primary treatment available for people who use MA, and also appears to have the most solid evidence base. ${ }^{29} 30$ Contingency management (CM) has been shown to be an efficacious psychological treatment for people who use $\mathrm{MA}^{31}$ and has increased MA abstinence and decreased MA-related risk behaviour. ${ }^{53} 33$ Certain psychological interventions, such as cognitive behavioural therapy (CBT) (including the Matrix Model), and approaches, such as Motivational Interviewing, ${ }^{27}$ have been effective in reducing MA use and reducing depressive symptoms in people who use MA. ${ }^{29}$ These interventions have also been associated with positive mental health outcomes, such as improved well-being, following treatment in people who use MA. ${ }^{34}$ However, it remains unclear as to what psychological interventions provide the most solid evidence base for reducing MA use and symptoms of mental ill health. As mental health symptoms are increasingly common and increasing in severity among people who use MA, there is a requirement for research on the efficacy of psychological interventions for MA use and mental health symptomatology.

\section{Why is it important to do this review?}

Further clarity regarding evidence for the efficacy of psychological treatments for MA use and co-occurring mental health symptoms or conditions is necessary. A Cochrane review conducted by Minozzi et al ${ }^{35}$ researched psychological interventions for psychostimulant use and covered a broad range of psychostimulants, including amphetamine-type stimulants, MDMA and cocaine. However, that review did not focus on MA and mental health outcomes, and only included two studies measuring depression and MA use. A review by Shoptaw et $a \hat{l}^{6}$ focused on treatment for amphetamine withdrawal, incorporating psychological and pharmacological treatment, and primarily focused on the withdrawal syndrome when using amphetamines. Another review by Shoptaw et $a \hat{l}^{37}$ focused on pharmacological treatment for amphetamines and psychosis. Cochrane reviews have assessed treatment for MA withdrawal and found no effective medication approaches. ${ }^{27} 36$

Hellem $e t a l^{4}$ conducted a review on MA and coexisting depressive symptoms, reviewing nine studies incorporating psychological intervention only; psychological 
combined with pharmacological interventions; and pharmacological only, and found no research supporting one single treatment approach over others on either MA or depression outcomes. Overall, it appears that psychological therapies remain the most effective treatment option for MA use, and that pharmacotherapies may be used as an adjunct. ${ }^{21}$ As it is difficult to maintain enduring behaviour changes in people who experience problems with drug use, ${ }^{29}$ the longevity of treatment effects relating to long-term abstinence and a range of mental health symptoms or conditions should be further assessed..$^{38} 39$

Cochrane reviews conducted thus far have not focused on psychological treatment for MA and co-occurring symptoms of mental ill health. ${ }^{35} 3740$ The present review will focus on studies which have measured MA use and mental health symptomatology or diagnoses at baseline and post-treatment. It will report on MA use outcomes, MA use and mental health symptom outcomes, and MA use and coexisting mental disorders. A review of this kind will contribute to the literature by highlighting psychological interventions that can reduce the global public health burden of disease in terms of MA use and mental health outcomes. ${ }^{41}$ Thus, this review will assess the effectiveness of psychological treatments in reducing MA use and associated symptoms of mental ill health. ${ }^{41}$ Results will assist public health and clinical use of treatment for coexisting MA use and mental health symptoms or conditions. ${ }^{42}$ This systematic review represents an important step in summarising the available evidence for psychological treatment for MA use and will allow for identification of areas for future research.

\section{Objectives}

Employing studies of psychological treatment of MA use which measure MA use and mental health symptoms or mental disorders at baseline and post-treatment, the aims of this review are to

1. examine the effectiveness of psychological treatments in reducing MA use and/or increasing abstinence rates among people who use MA;

2. examine the effectiveness of psychological treatments for coexisting mental health symptoms or mental disorders among people who use MA;

3. conduct secondary analyses examining other outcomes following psychological treatment (bloodborne virus (BBV) risk behaviour, other substance use, treatment engagement, retention, physical activity, quality of life, global assessment of functioning) and potential mediators;

4. identify future research directions.

\section{Review questions}

For adults using MA:

1. Does psychological treatment change (reduce/ increase) symptoms of mental ill health or coexisting mental disorders?

2. What psychological treatments are effective in reducing MA use and/or increasing abstinence rates?
3. What psychological treatments are effective in changing (reducing/increasing) symptoms of mental ill health or coexisting mental disorders?

4. Is the effectiveness of psychological treatment for MA use influenced by treatment engagement (quantity, frequency and/or duration of therapy attendance)?

\section{METHODS AND ANALYSIS}

A systematic review will be conducted using the Preferred Reporting Items for Systematic Review and Meta-Analysis (PRISMA) guidelines. ${ }^{43}$

\section{Eligibility criteria}

Types of studies

This systematic review is focused on the efficacy of psychological interventions for reducing MA use. Studies included will report (1) MA use and (2) mental health symptoms and/or disorders at baseline and post-treatment. Controlled trials such as randomised controlled trials (RCTs, cluster and parallel design) will be eligible. Case controls, crossover trials, one-arm trials, non-randomised trials, cross-sectional studies and cohort studies will be excluded.

\section{Types of participants}

Participants included in the review will be adults (over 18 years), using MA alone or in combination with other substances (polydrug use). Participants may be in an inpatient unit (drug and alcohol rehabilitation or hospital setting), residing in the community, engaging in psychotherapy or inmates in a prison setting.

\section{Types of interventions}

Psychological interventions of interest include behaviour therapy, CM, CBT, the community reinforcement approach, acceptance and commitment therapy, dialectical behaviour therapy, motivational interviewing, psychotherapy, group therapy, mutual aid (narcotics anonymous/SMART recovery) and residential treatment. Web-based, telephone and smartphone delivered interventions will also be included. Interventions delivered in any setting will be included (eg, private practice, hospital, rehabilitation and residential treatment centre). Psychological interventions must include one or more psychological strategies designed to modify behaviour.

\section{Types of comparison conditions}

Interventions may be compared with active controls (eg, psychological interventions and 12-step programmes), treatment as usual (TAU) and/or inactive controls (eg, wait-list control or standard care). Interventions can be of any duration, delivery, frequency and intensity.

\section{Types of outcome measures}

\section{Primary outcomes}

1. Any outcome measure reporting change (reduction/ increase) or abstinence in MA use following psychological treatment for MA use. 
2. Any outcome measure reporting change (reduction/ increase) in mental health symptoms or diagnoses following psychological treatment for MA use.

\section{Secondary outcomes}

1. Change in other drug use following psychological treatment. Reduction/increase or abstinence in drug use (tobacco, amphetamine-type stimulants, alcohol, cannabis, cocaine, opioids, tranquillizers or polydrug use) following treatment.

2. Treatment engagement and retention in psychological treatment for MA use.

3. BBV risk reduction (injecting drugs/sexual risk behaviour) following psychological treatment for MA use.

4. Change in physical health following treatment.

5. Change in aspects of quality of life following treatment.

6. Difference in levels of functioning pretreatment and post-treatment (global assessment of functioning or social functioning).

Outcomes reflect any time frame (eg, short-term and long-term) and can be rated by clients or clinicians, in the form of an assessment by objective or subjective measures (eg, questionnaire, monitoring form, urine and blood).

\section{Information sources}

\section{Search strategy}

The search strategy will follow the Cochrane Handbook for Systematic Reviews of Interventions. In June 2016, we consulted with a qualified librarian and identified relevant scientific electronic databases (MEDLINE, EMBASE, CINAHL, PsychINFO, Scopus). Registration databases will also be searched (Cochrane Central Register of Clinical Trials, US government website of clinic trials and WHO International Clinical Trials Registry).

Search terms were developed from existing reviews to cover psychological interventions ${ }^{242744}$ (eg, CBT and $\mathrm{CM}$ ) and MA (see online supplementary appendix A). The MEDLINE expert search for RCTs will be used to identify RCTs. Subject headings, titles, keywords and abstracts specific to each of the identified databases will be recognised and subject headings will be exploded to allow narrower terms to be included where possible. Publications will be limited to human studies. No limits will be placed on publication year. Publications must be available in English to be included, but any non-English language publications that are eligible on the basis of a translated abstract will be noted. Reference lists of publications and extra studies not identified by the original search will be hand searched to identify any further publications. All publications will be organised using Endnote. Prior to final analyses, searches will be rerun and further studies will be retrieved for inclusion.

\section{Classification of studies}

Titles and abstracts will be identified by AS and a second reviewer using the following three-step process.
Step 1: identification of studies for exclusion

Step 1 will involve identifying studies for exclusion. Titles and abstracts will be reviewed and excluded if articles are (1) not peer-reviewed journal articles, (2) duplicates, (3) do not use a controlled design, (4) do not include a psychological intervention or (5) do not include relevant behavioural change outcome measures associated with MA use or mental health outcomes.

\section{Step 2: classification of studies}

Step 2 will involve classification of studies, in which full text and/or abstracts of any remaining studies will be examined to identify studies to be evaluated, including reviews (summaries, descriptive, critical and/or systematic reviews) and controlled studies.

\section{Step 3: cross-checking}

Step 3 will involve cross-checking publications found in previous steps to check for eligibility and reclassified if necessary. In case of disagreement between reviewers, the final classification will be made by consensus, with the involvement of a third reviewer (ALB). Articles excluded in Step 1 will not be cross-checked because they will not be relevant. Studies identified in Step 2 will be retained for further examination.

\section{Data extraction from evaluation studies}

Data extraction will be performed by AS and a second reviewer. Before using the extraction form, it will be piloted on several studies and adapted as needed. When duplicate reports of the same study are identified (eg, conference abstracts or associated journal articles), data from each report will be extracted independently and then combined across multiple data collection forms. In accordance with Cochrane guidelines, methodological critique and assessment of risk of bias will be conducted individually by two raters and judgements reached by consensus. If a disagreement occurs, a third independent rater will establish final ratings made via consensus. The occurrence and resolution of any disagreements will be recorded to allow for the assessment of reliability of coding. If there are not enough details of trials reported, then authors of studies will be contacted.

To ensure functionality, extraction forms will be pretested in $10 \%$ of the identified articles. The Cochrane Handbook for Systematic Reviews ${ }^{45}$ will be used to guide data extraction. Data extraction will include:

1. Participant information: $\mathrm{n}$ values at each stage of the study (at baseline and follow-up, and include reasons for non-participation), eligibility criteria, treatment setting, descriptive data, diagnostic criteria and treatment history.

2. Methods of each study: design and setting (country), methodological limitations reported and observed (eg, recruitment allocation and data collection methods, blinding, comparability of groups at baseline, appropriateness of analysis). 
3. Type of interventions: duration of treatment (number of sessions), number of groups, type of sessions (group/individual), method of delivery and description of control intervention(s).

4. Primary and secondary outcomes: percentage of treatment sessions attended, data collection sources/ methods, process measures/mediators/mechanisms, economic outcomes, satisfaction-related outcomes and follow-up period.

5. Results of studies: primary (change in MA use and mental health outcomes) and secondary outcomes (BBV risk behaviour, other substance use, treatment engagement, retention, physical activity, quality of life and global assessment of functioning).

\section{Methodological critique of evaluation research}

Review authors will perform risk of bias assessments and methodological critique independently (AS and second reviewer), and a third person will resolve discrepancies $(\mathrm{AB})$. If disagreement occurs, final ratings will be made via consensus, following discussion with coauthors. ${ }^{46}$ The 'Cochrane Collaboration's Risk of Bias' tool will be used to measure risk of bias ${ }^{45}$ with items judged as low, high or unclear risk. A 'high' or 'unclear' risk of bias will be deemed by allocation concealment and selection bias, as these factors have been suggested to be sources of bias from previous research. ${ }^{45}$

\section{Grading the strength of evidence}

The overall quality of evidence on outcomes will be presented using the GRADE (Grades of Recommendation, Assessment, Development and Evaluation) ${ }^{45}$ approach. This involves consideration of within study risk of bias, heterogeneity, directness of evidence, precision of effect estimates and risk of publication bias. The overall quality of evidence will be rated at four levels: high, moderate, low and very low.

\section{Measures of treatment effect}

A quantitative synthesis of the outcomes from the included studies will be reported, using a meta-analysis if possible. If a meta-analysis or quantitative synthesis is not possible for some studies (due to lack of comparable interventions or outcome measures), a narrative synthesis of the findings will be used to report outcomes. This synthesis will be formatted around intervention content and type, population features and outcomes. The context of treatment (eg, psychological versus active control) and the type of outcomes will be described. Where possible, 'summary of findings' (SOF) tables will be created for each comparison (eg, psychological intervention versus TAU). These tables will provide key information regarding evidence quality, a summary of available data on outcome variables and the degree of the effect of interventions.

\section{Dichotomous outcome measures}

Risk ratios (RR) will be used to measure dichotomous outcome measures, ${ }^{47}$ where a $95 \%$ CI will be provided for the primary outcome of each trial.

\section{Continuous outcome measures}

Continuous outcome measures will be measured by Cohen's $d$ to calculate effect sizes. A small effect size will be considered as $0.2-0.49$, a moderate effect size is $0.5-0.79$ and a large effect size is greater than $0.8 .^{48}$ If sample sizes are small, Hedge's $g$ will be used in place of $d$.

\section{Ethics and dissemination}

As data have already been published and analysis is secondary, no ethics approval is required. Findings of this systematic review will be presented for peer review in an appropriate journal. Findings will be presented to researchers and clinicians at suitable conferences.

\section{Amendments}

If the protocol needs to be amended, the date of each amendment, the change and the rationale will be described in this section. The search strategy was amended on the 26th of July 2017 following consultation with a research librarian. This was following revision of the previous search strategy created in 2016, to ensure clarity and to make the search more robust in nature. The new search ensures that both the relevant MESH headings as well as keyword searches are used. Additionally, correct truncation was applied, and adjacency searches standardised across each of the searches.

Contributors AS is the guarantor of the review. AS, ALB, AD, AMJD, KM and $\mathrm{JB}$ assisted in writing the protocol. AS performed the preliminary searches, will perform data extraction, conduct quality assessments and draft the systematic review paper. AMJD will screen references and cross-check data extraction and perform independent quality ratings. AS developed the search strategy with the assistance of a research librarian. KC provided statistical expertise. ALB provided expertise on psychological treatment for MA use. AD provided expertise on pharmacotherapy. JB provided expertise on the process of systematic reviews. All other authors contributed to the conception and design of this systematic review and will assist AS and AMJD to resolve any discrepancies in relation to data extraction, study inclusion and quality ratings. AS, ALB, AD, AMJD, JB, KM and NL read, provided feedback and approved the protocol manuscript and will offer critical revisions for the review manuscript.

Funding The School of Psychology and the School of Medicine and Public Health, University of Newcastle, NSW, Australia supported this work. No specific grant from any funding agency in the commercial, public or not-for-profit sectors was received for this research.

Competing interests None declared.

Patient consent Not applicable.

Provenance and peer review Not commissioned; externally peer reviewed.

Open Access This is an Open Access article distributed in accordance with the Creative Commons Attribution Non Commercial (CC BY-NC 4.0) license, which permits others to distribute, remix, adapt, build upon this work non-commercially, and license their derivative works on different terms, provided the original work is properly cited and the use is non-commercial. See: http://creativecommons.org/ licenses/by-nc/4.0/

(c) Article author(s) (or their employer(s) unless otherwise stated in the text of the article) 2017. All rights reserved. No commercial use is permitted unless otherwise expressly granted. 


\section{REFERENCES}

1. Suvanchot KS, Somrongthong R, Phukhao D. Efficacy of group motivational interviewing plus brief cognitive behavior therapy for relapse in amphetamine users with co-occurring psychological problems at Southern Psychiatric Hospital in Thailand. J Med Assoc Thai 2012:95:1075-80.

2. Colfax G, Santos GM, Chu P, et al. Amphetamine-group substances and HIV. Lancet 2010;376:458-74.

3. Johnson BA, Roache JD, Ait-Daoud N, et al. Effects of isradipine on methamphetamine-induced changes in attentional and perceptualmotor skills of cognition. Psychopharmacology 2005;178(23):296-302

4. Hellem TL, Lundberg KJ, Renshaw PF. A review of treatment options for co-occurring methamphetamine use disorders and depression. $J$ Addict Nurs 2015;26:14-23.

5. Roll JM, Petry NM, Stitzer ML, et al. Contingency management for the treatment of methamphetamine use disorders. Am J Psychiatry 2006;163:1993-9.

6. Cadet JL, Jayanthi S, Deng X. Speed kills: cellular and molecular bases of methamphetamine-induced nerve terminal degeneration and neuronal apoptosis. Faseb J 2003;17:1775-88.

7. Degenhardt L, Sara G, McKetin R, et al. Crystalline methamphetamine use and methamphetamine-related harms in Australia. Drug Alcohol Rev 2017;36.

8. APA. Diagnostic and statistical manual of mental disorders. 5th ed. Arlington, VA: American Psychiatric Association, 2013.

9. Global Burden of Disease Study 2013 Collaborators. Global, regional, and national incidence, prevalence, and years lived with disability for 301 acute and chronic diseases and injuries in 188 countries, 19902013: a systematic analysis for the Global Burden of Disease Study 2013. Lancet 2015;386:743-800.

10. Chomchai $\mathrm{C}$, Chomchai S. Global patterns of methamphetamine use. Curr Opin Psychiatry 2015;28:269-74.

11. Degenhardt L, Hall W. Extent of illicit drug use and dependence, and their contribution to the global burden of disease. Lancet 2012;379:55-70.

12. AlHW. National Drug Strategy Household Survey Report. Canberra: ACT, 2013.

13. NSW Health. Crystalline methamphetamine. background paper. 2015. NSW Government (accessed 20 Aug 2015).

14. Darke S, Kaye S, McKetin R, et al. Major physical and psychological harms of methamphetamine use. Drug Alcohol Rev 2008;27:253-62.

15. Joe GW, Rowan-Szal GA, Greener JM, et al. Male methamphetamine-user inmates in prison treatment: duringtreatment outcomes. J Subst Abuse Treat 2010;38:141-52.

16. Fletcher JB, Reback CJ. Antisocial personality disorder predicts methamphetamine treatment outcomes in homeless, substancedependent men who have sex with men. J Subst Abuse Treat 2013;45:266-72.

17. McKetin R, Kelly E, McLaren J. The relationship between crystalline methamphetamine use and methamphetamine dependence. Drug Alcohol Depend 2006;85:198-204.

18. Glasner-Edwards S, Mooney LJ, Marinelli-Casey P, et al. Psychopathology in methamphetamine-dependent adults 3 years after treatment. Drug Alcohol Rev 2010;29:12-20.

19. Newton TF, De La Garza R, Kalechstein AD, et al. Theories of addiction: methamphetamine users' explanations for continuing drug use and relapse. Am J Addict 2009;18:294-300.

20. Kay-Lambkin FJ, Baker AL, McKetin R, et al. Stepping through treatment: reflections on an adaptive treatment strategy among methamphetamine users with depression. Drug Alcohol Rev 2010;29:475-82.

21. Rose ME, Grant JE. Pharmacotherapy for methamphetamine dependence: a review of the pathophysiology of methamphetamine addiction and the theoretical basis and efficacy of pharmacotherapeutic interventions. Ann Clin Psychiatry 2008;20:145-55.

22. Karila L, Weinstein A, Aubin HJ, et al. Pharmacological approaches to methamphetamine dependence: a focused review. $\mathrm{Br} \mathrm{J}$ Clin Pharmacol 2010;69:578-92.

23. Darke S, Farrell M. Which medications are suitable for agonist drug maintenance? Addiction 2016;111:767-74.
24. Lile JA, Stoops WW, Glaser PE, et al. Physiological and subjective effects of acute intranasal methamphetamine during extended-release alprazolam maintenance. Drug Alcohol Depend 2011;119:187-93.

25. Elkashef AM, Rawson RA, Anderson AL, et al. Bupropion for the treatment of methamphetamine dependence. Neuropsychopharmacology 2008;33:1162-70.

26. Anderson AL, Li SH, Markova D, et al. Bupropion for the treatment of methamphetamine dependence in non-daily users: a randomized double-blind, placebo-controlled trial. Drug Alcohol Depend 2015;150:170-4

27. Ciketic S, Hayatbakhsh MR, Doran CM, et al. A review of psychological and pharmacological treatment options for methamphetamine dependence. J Subst Use 2012;17:363-83.

28. Degenhardt L, Roxburgh A, Black E, et al. The epidemiology of methamphetamine use and harm in Australia. Drug Alcohol Rev 2008;27:243-52.

29. Rawson RA, Marinelli-Casey P, Anglin MD, et al. A multi-site comparison of psychosocial approaches for the treatment of methamphetamine dependence. Addiction 2004;99:708-17.

30. Wisdom JP, Manuel JI, Drake RE. Substance use disorder among people with first-episode psychosis: a systematic review of course and treatment. Psychiatr Serv 2011;62:1007-12.

31. Baker A, Lee NK, Claire M, et al. Brief cognitive behavioural interventions for regular amphetamine users: a step in the right direction. Addiction 2005;100:367-78.

32. Reback CJ, Peck JA, Fletcher JB, et al. Lifetime substance use and HIV sexual risk behaviors predict treatment response to contingency management among homeless, substance-dependent MSM. $J$ Psychoactive Drugs 2012;44:166-72.

33. Ling Murtaugh K, Krishnamurti T, Davis AL, et al. Spend today, clean tomorrow: predicting methamphetamine abstinence in a randomized controlled trial. Health Psychol 2013;32:958-66.

34. Baker A, Bucci S, Lewin TJ, et al. Cognitive-behavioural therapy for substance use disorders in people with psychotic disorders: randomised controlled trial. Br J Psychiatry 2006;188:439-48.

35. Minozzi S, Saulle R, De Crescenzo F, et al. Psychosocial interventions for psychostimulant misuse. Cochrane Database Syst Rev 2016;9:CD011866.

36. Shoptaw SJ, Kao U, Heinzerling K, et al. Treatment for amphetamine withdrawal. Cochrane Database Syst Rev 2009;2:CD003021.

37. Shoptaw SJ, Kao U, Ling W. Treatment for amphetamine psychosis. Cochrane Database Syst Rev 2009;1:CD003026.

38. Lee NK, Rawson RA. A systematic review of cognitive and behavioural therapies for methamphetamine dependence. Drug Alcohol Rev 2008;27:309-17.

39. Tait RJ, McKetin R, Kay-Lambkin F, et al. Six-month outcomes of a web-based intervention for users of amphetamine-type stimulants: randomized controlled trial. J Med Internet Res 2015;17:e105.

40. Shoptaw SJ, Kao U, Heinzerling K, et al. Treatment for amphetamine withdrawal. Cochrane Database Syst Rev 2009;2:CD003021.

41. Sullivan D, McDonough M. Methamphetamine: where will the stampede take us? J Law Med 2015;23:41-9.

42. Glasner-Edwards S, Mooney LJ, Marinelli-Casey P, et al. Clinical course and outcomes of methamphetamine-dependent adults with psychosis. J Subst Abuse Treat 2008;35:445-50.

43. Moher D, Liberati A, Tetzlaff J, et al. Preferred reporting items for systematic reviews and meta-analyses: the PRISMA statement. Int $J$ Surg 2010;8:336-41.

44. Knapp WPS, Farrell, M B, Silva de Lima M. Psychosocial interventions for cocaine and psychostimulant amphetamines related disorders (Review). Cochrane Database Syst Rev 2007;18:CD003023.

45. Higgins JPT, Green S. Cochrane Handbook for Systematic Reviews of Interventions. The Cochrane Collaboration, 2011.

46. Moseley AM, Herbert RD, Sherrington C, et al. Evidence for physiotherapy practice: a survey of the physiotherapy evidence database (PEDro). Aust J Physiother 2002;48:43-9.

47. Boissel JP, Cucherat M, Gueffier F, et al. Methodology outline of the problem of indices of therapeutic efficacy. II the Indices. Therapie 1999:54:309-14.

48. Cohen J. A power primer. Psychol Bull 1992;112:155-9. 